\title{
Particles Collision near Kerr-Sen Dilaton-Axion Black Hole
}

\author{
Ujjal Debnath* \\ Department of Mathematics, Indian Institute of Engineering Science and Technology, \\ Shibpur, Howrah-711 103, India.
}

August 19, 2018

\begin{abstract}
Here we consider static, axially symmetric, rotating and charged Kerr-Sen Dilaton-Axion black hole metric in generalized Boyer-Lindquist coordinates as particle accelerators. We obtain the geodesic motions of particle. We find the effective potential and discuss the circular orbit of a particle. We investigate the center of mass energy of two colliding neutral particles with different rest masses falling from rest at infinity to near the non-extremal horizons (event horizon and Cauchy horizon) and extremal horizon of the Kerr-Sen black hole. Analogous to the Compton process, we discuss the collision of a particle and a massless photon. Finally, we find the center of mass energy due to the collision of two photons in the background of Kerr-Sen black hole.
\end{abstract}

Keywords: Black hole, Particles collision, Center of mass energy.

\section{Introduction}

Due to astrophysical research, Bañados, Silk and West (BSW) [1 have proposed the mechanism for the collision of two particles falling from rest at infinity into the horizons of Kerr black hole and determined that the center of mass $(\mathrm{CM})$ energy in the equatorial plane $(\theta=\pi / 2)$ may be high in the extremal black hole. Lake [2] found that the CM energy of the particles diverges to infinity at the inner horizon of non-extremal Kerr black hole. Further, Wei et al [3] investigated that the CM energy of the collision of the particles around Kerr-Newmann black hole depends on the spin $(a)$ and charge $(Q)$ parameters. A general review of the collision mechanism is found in ref [4]. After that Liu et al [5] demonstrated the CM energy of the collision of particles near the horizons of Kerr-Taub-NUT black hole and then Zakria et al [6] investigated the CM energy of the collision for two neutral particles with different rest masses in the background of a Kerr-NewmanTaub-NUT black hole. Sharif et al [7] found the CM energy of the collision of the particles near the horizons of the most general accelerating Plebanski-Demianski black hole and shown that the CM energy depends on the accelerating parameter and other parameters also. Particle acceleration in charged dilaton black hole has been investigated in ref [8]. The authors in [9] studied the particle accelerations and collisions in the back ground of a cylindrical black holes.

*Email: ujjaldebnath@gmail.com ; ujjal@iucaa.ernet.in 
On the other hand, Abhas et al [10] studied the geodesic study of regular Hayward black hole and its rotating version has been discussed by Bambi et al [11]. The particle acceleration near the regular Hayward and Bardeen black holes has been analyzed by Pradhan [12. Also the CM energy of the collision for charged particles near the Bardeen black hole has been analyzed [13. After that it was demonstrated the CM energy of the collision of two particles near horizon of the rotating Hayward's black hole [14] and rotating Bardeen black hole [15]. Recently we have also found the CM energy for the rotating versions of modified Hayward and Bardeen black hole [16]. Tursunov et al [17] have studied the particle accelerations and collisions of black string. Fernando [18] has studied the possibility of high CM energy of two particles colliding near the infinite red-shift surface of a charged BH in string theory. Till now, several authors [19, 20, 21, 22, 23, 24, 25, 26, 27, 28, 29, 30, 31, 32, 34, 35, 36] have studied the CM energy of the collision of particles near the horizons of black holes. Our main motivation is to investigate the geodesic study of the static, axially symmetric, rotating and charged Kerr-Sen Dilaton-Axion black hole [37. The CM energy and the particles orbit are investigated for two colliding neutral particles of different rest masses falling from infinity to near the event horizon, Cauchy horizon and extremal horizon of Kerr-Sen Dilaton-Axion black hole. Also the collision of a particle with massless photon and photon-photon collision near Kerr-Sen black hole are discussed and the concerned CM energy are calculated. Finally we conclude the results for particle acceleration near the Kerr-Sen DilatonAxion black hole.

\section{Equations of motion for a particle near Kerr-Sen Dilaton-Axion Black Hole}

In 1992, Sen [38] has constructed a solution of the classical equations of motion arising in the low-energy effective field theory for heterotic string theory. This low energy heterotic string black hole carries a finite amount of charge, angular momentum and magnetic dipole moment. It could be produced by twisting method and starting from a rotating Kerr black hole having no charge. So, sometimes it is called twisted Kerr black hole or Kerr-Sen black hole. In Kerr-Sen black hole, the thermodynamic properties are discussed in [39, 40] and analytical gravitational lensing are discussed in [37, 41]. Hidden symmetries, null geodesics and photon capture in the Sen black hole have been studied in [42. Thermodynamic and astrophysical consequences of the Sen black hole have been studied by several authors [43, 44, 45, 46, 47, 48, 49, 50, 51. Here we consider static, axially symmetric, rotating and charged Kerr-Sen Dilaton-Axion black hole metric in generalized Boyer-Lindquist coordinates given by [37]

$$
\begin{gathered}
d s^{2}=-\left(1-\frac{2 M r}{\rho^{2}(r)}\right) d t^{2}+\frac{\rho^{2}(r)}{\Delta(r)} d r^{2}+\rho^{2}(r) d \theta^{2}-\frac{4 M r a \sin ^{2} \theta}{\rho^{2}(r)} d t d \phi \\
+\left(r\left(r+r_{\alpha}\right)+a^{2}+\frac{2 M r a^{2} \sin ^{2} \theta}{\rho^{2}(r)}\right) \sin ^{2} \theta d \phi^{2}
\end{gathered}
$$

where

$$
\begin{gathered}
\Delta(r)=r\left(r+r_{\alpha}\right)-2 M r+a^{2}, \\
\rho^{2}(r)=r\left(r+r_{\alpha}\right)+a^{2} \cos ^{2} \theta
\end{gathered}
$$


Here $M$ is the mass of the black hole, $a$ is the angular momentum per unit mass of the black hole $(=J / M)$ and $r_{\alpha}=\frac{Q^{2}}{M}$, where $Q$ is the charge of the black hole. From the Kerr-Sen black hole metric, we observe that there is no naked singularity. For $a=0$, the Kerr-Sen black hole reduces to the Gibbons-Maeda-Garfinkle-Horowitz-Strominger (GMGHS) black hole and for $r_{\alpha}=0$, we get Kerr black hole. Further if both $a=0$ and $r_{\alpha}=0$ then it reduces to Schwarzschild black hole. The horizon found by setting $\Delta(r)=0$, i.e.,

$$
r_{ \pm}=\left(M-\frac{Q^{2}}{2 M}\right) \pm \sqrt{\left(M-\frac{Q^{2}}{2 M}\right)^{2}-a^{2}}
$$

Here $r_{+}$is the outer/event horizon and $r_{-}$is the inner/Cauchy horizon. The horizons exist if $a \leq M-\frac{Q^{2}}{2 M}$. The extremal limit of Kerr-Sen black hole describes $r_{+}=r_{-}=a=M-\frac{Q^{2}}{2 M}$. The ergosphere occurs when coefficient of $d t^{2}$ is zero i.e., $g_{t t}=0$. So the radius of the ergosphere is given by

$$
r_{\text {ergo } \pm}=\left(M-\frac{Q^{2}}{2 M}\right) \pm \sqrt{\left(M-\frac{Q^{2}}{2 M}\right)^{2}-a^{2} \cos ^{2} \theta}
$$

Since $\cos ^{2} \theta<1$, so we have $r_{\text {ergo+ }}>r_{+}$and $r_{\text {ergo- }}<r_{-}$. Thus the ergospheres occurs inside the Cauchy horizon or outside the event horizon. The angular momentum $\Omega$ at the horizon is obtained by the killing vector $\left(\partial_{t}+\Omega \partial_{\phi}\right)$ is null at the horizon [38] i.e.,

$$
g_{t t}+2 g_{t \phi} \Omega+g_{\phi \phi} \Omega^{2}=0
$$

which simplifies to

$$
\Omega_{ \pm} \equiv \Omega=\frac{a}{2 M r_{ \pm}}=\frac{1}{2 M a}\left[\left(M-\frac{Q^{2}}{2 M}\right) \mp \sqrt{\left(M-\frac{Q^{2}}{2 M}\right)^{2}-a^{2}}\right]
$$

Since $r_{+}>r_{-}$, so we have $\Omega_{+}<\Omega_{-}$. Thus the angular momentum on the Cauchy horizon is always larger than the event horizon. Now consider a particle exhibits the geodesic motion in the Kerr-Sen Dilaton-Axion spacetime. Let $U^{a}=\left(U^{t}, U^{r}, U^{\theta}, U^{\phi}\right)$ be the four velocity of the particle. On the equatorial motion of the particle, $\theta=\pi / 2$, so $U^{\theta}=0$. Thus the metric admits only two killing vectors $\partial_{t}$ and $\partial_{\phi}$. Using these killing vectors, the energy $E$ and angular momentum $L$ can be defined by [7, 36]

$$
E=-g_{a b}\left(\partial_{t}\right)^{a} U^{b}=-g_{t t} U^{t}-g_{t \phi} U^{\phi}
$$

and

$$
L=g_{a b}\left(\partial_{\phi}\right)^{a} U^{b}=g_{t \phi} U^{t}+g_{\phi \phi} U^{\phi}
$$

From these, we obtain the 4 velocity vector along $t$ and $\phi$ directions as in the following forms:

$$
U^{t}=\frac{1}{\Delta(r)}\left[A(r) E-\frac{2 M a}{r+r_{\alpha}} L\right]
$$

and

$$
U^{\phi}=\frac{1}{\Delta(r)}\left[\frac{2 M a}{r+r_{\alpha}} E+\left(1-\frac{2 M}{r+r_{\alpha}}\right) L\right]
$$


where

$$
A(r)=r\left(r+r_{\alpha}\right)+a^{2}+\frac{2 M a^{2}}{r+r_{\alpha}} \geq 0
$$

On the horizon $r_{ \pm}$, we must have $A\left(r_{ \pm}\right)=4 M^{2} r_{ \pm}$. Now use the normalizing condition:

$$
g_{a b} U^{a} U^{b}=\epsilon
$$

where $\epsilon=-1$ represents the timelike geodesic, $\epsilon=0$ represents the null geodesic and $\epsilon=+1$ represents spacelike geodesic. Now we obtain the radial component of the 4-velocity as

$$
U^{r}= \pm \frac{1}{\sqrt{g_{r r}}}\left[\epsilon-g_{t t}\left(U^{t}\right)^{2}-2 g_{t \phi} U^{t} U^{\phi}-g_{\phi \phi}\left(U^{\phi}\right)^{2}\right]^{\frac{1}{2}}
$$

which yields

$$
U^{r}= \pm \frac{1}{\sqrt{r\left(r+r_{\alpha}\right)}}\left[A(r) E^{2}-\frac{4 M a}{r+r_{\alpha}} E L-\left(1-\frac{2 M}{r+r_{\alpha}}\right) L^{2}+\epsilon \Delta(r)\right]^{\frac{1}{2}}
$$

where \pm indicate the radially outgoing and incoming geodesics respectively. Since the timelike component of the 4 -velocity $\left(U^{t}\right)$ is greater than zero (causally connected), so we get

$$
E\left(r\left(r+r_{\alpha}\right)+a^{2}+\frac{2 M a^{2}}{r+r_{\alpha}}\right) \geq \frac{2 L M a}{r+r_{\alpha}}
$$

On the horizon $(\Delta(r)=0)$, the above inequality implies

$$
L \leq \frac{2 M r E}{a}=\frac{E}{\Omega}
$$

The upper bound of the specific angular momentum $(E / \Omega)$ at the horizon of the Kerr-Sen black hole is known as the critical angular momentum and denoted by $L_{c}$ and so $L_{c}=\frac{2 M r E}{a}$ where $r$ satisfies $\Delta(r)=0$. Now the effective potential can be determined by equation

$$
\frac{1}{2}\left(U^{r}\right)^{2}+V_{e f f}(r)=\frac{1}{2}\left(E^{2}+\epsilon\right)
$$

where

$$
V_{\text {eff }}(r)=\frac{1}{2}\left(E^{2}+\epsilon\right)-\frac{1}{2 r\left(r+r_{\alpha}\right)}\left[A(r) E^{2}-\frac{4 M a}{r+r_{\alpha}} E L-\left(1-\frac{2 M}{r+r_{\alpha}}\right) L^{2}+\epsilon \Delta(r)\right]
$$

We observe that $V_{\text {eff }}(r) \rightarrow 0$ as $r \rightarrow \infty$ but $V_{\text {eff }}(r)$ diverges near the center $(r=0)$ of the Kerr-Sen black hole. Assume that the radius of circular orbit is $r=r_{c}$. So the conditions for the circular orbit are given by

$$
V_{e f f}\left(r_{c}\right)=0, \quad\left[\frac{d V_{e f f}(r)}{d r}\right]_{r=r_{c}}=0
$$

which implies two equations

$$
A\left(r_{c}\right) E^{2}-\frac{4 M a}{r_{c}+r_{\alpha}} E L-\left(1-\frac{2 M}{r_{c}+r_{\alpha}}\right) L^{2}+\epsilon \Delta\left(r_{c}\right)-r\left(r+r_{c}\right)\left(E^{2}+\epsilon\right)=0
$$


and

$$
\begin{gathered}
4 a M\left(3 r_{c}+r_{\alpha}\right) E L+\left(\left(r_{c}+r_{\alpha}\right)\left(2 r_{c}+r_{\alpha}\right)-2 M\left(3 r_{c}+r_{\alpha}\right)\right) L^{2}+r_{c}\left(r_{c}+r_{\alpha}\right)^{2}\left(A^{\prime}\left(r_{c}\right) E^{2}+\epsilon \Delta^{\prime}\left(r_{c}\right)\right) \\
-\left(r_{c}+r_{\alpha}\right)\left(2 r_{c}+r_{\alpha}\right)\left(A\left(r_{c}\right) E^{2}-\epsilon \Delta\left(r_{c}\right)\right)=0
\end{gathered}
$$

From these equations, we may obtain

$$
\begin{gathered}
a M\left[a^{2}+\left(r_{c}+r_{\alpha}\right)\left(3 r_{c}+r_{\alpha}\right)\right] E L+\left[-4 a^{2} M+\left(r_{c}+r_{\alpha}\right)\left\{\left(2 r_{c}+r_{\alpha}\right)\left(r_{c}+r_{\alpha}\right)-2 M\left(3 r_{c}+r_{\alpha}\right)\right\}\right] L^{2} \\
+2 \epsilon M\left[2 a^{4}+r_{c}^{2}\left(r_{c}+r_{\alpha}\right)^{2}+a^{2}\left(r_{c}^{2}-r_{\alpha}^{2}\right)-4 a^{2} M\left(2 r_{c}+r_{\alpha}\right)\right]=0
\end{gathered}
$$

From the above equation we may obtain the radius $\left(r_{c}\right)$ of the circular orbit of a particle near the Kerr-Sen black hole.

\section{Center of Mass energy for two neutral particles near horizons}

We now determine the center of mass energy (CME) for two colliding particles with rest masses $m_{1}$ and $m_{2}$ moving in the equatorial plane. The four-momentum of the $i$-th particle is given by [6]

$$
\left(p_{i}\right)^{a}=m_{i}\left(U_{i}\right)^{a}, \quad i=1,2, \quad a=t, r, \theta, \phi
$$

where $p_{i}$ is the four momentum of the $i$-th particle. The CME for two colliding particles is given by

$$
E_{c m}^{2}=-\left(p_{i}\right)^{a}\left(p_{i}\right)_{a}=-\left[m_{1}\left(U_{1}\right)^{a}+m_{2}\left(U_{2}\right)^{a}\right]\left[m_{1}\left(U_{1}\right)_{a}+m_{2}\left(U_{2}\right)_{a}\right]
$$

We assume the geodesic motion of the particles trajectories are timelike $(\epsilon=-1), g_{a b}\left(U_{i}\right)^{a}\left(U_{i}\right)^{b}=$ -1 , so the above equation can be written as

$$
\begin{aligned}
E_{c m}^{2} & =2 m_{1} m_{2}\left[\frac{\left(m_{1}-m_{2}\right)^{2}}{2 m_{1} m_{2}}+\left(1-g_{a b} U_{1}^{a} U_{2}^{b}\right)\right] \\
& =2 m_{1} m_{2}\left[\frac{\left(m_{1}-m_{2}\right)^{2}}{2 m_{1} m_{2}}+\left(1-g_{t t} U_{1}^{t} U_{2}^{t}-g_{t \phi} U_{1}^{t} U_{2}^{\phi}-g_{\phi t} U_{1}^{\phi} U_{2}^{t}-g_{\phi \phi} U_{1}^{\phi} U_{2}^{\phi}-g_{r r} U_{1}^{r} U_{2}^{r}\right)\right]
\end{aligned}
$$

which turns out to be

$$
\frac{E_{c m}}{\sqrt{2 m_{1} m_{2}}}=\left[\frac{\left(m_{1}-m_{2}\right)^{2}}{2 m_{1} m_{2}}+\frac{X(r)-\kappa Y(r)}{\Delta(r)}\right]^{\frac{1}{2}}
$$

where

$$
\begin{gathered}
X(r)=\left[A(r) E_{1} E_{2}-\frac{2 M a}{r+r_{\alpha}}\left(E_{1} L_{2}+E_{2} L_{1}\right)-\left(1-\frac{2 M}{r+r_{\alpha}}\right) L_{1} L_{2}+\Delta(r)\right], \\
Y(r)=\sqrt{Y_{1}(r) Y_{2}(r)}
\end{gathered}
$$

with

$$
Y_{i}(r)=\left[A(r) E_{i}^{2}-\frac{4 M a}{r+r_{\alpha}} E_{i} L_{i}-\left(1-\frac{2 M}{r+r_{\alpha}}\right) L_{i}^{2}-\Delta(r)\right], i=1,2
$$

Here $E_{i}$ and $L_{i}$ are respectively the specific energy and the specific angular momentum of the $i$-th particle $(i=1,2)$. We take $\kappa=+1$ when the particles move in the same direction and $\kappa=-1$ when the particles move in the opposite direction. We observe that the CME is invariant if we interchange the quantities $m_{1} \leftrightarrow m_{2}, E_{1} \leftrightarrow E_{2}$ and $L_{1} \leftrightarrow L_{2}$. We shall consider the collisions of particles near the non-extremal horizons (event horizon and Cauchy horizon) and extremal Kerr-Sen black hole and discuss the properties of CME. 


\subsection{Collision at non-extremal horizons}

On the non-extremal horizons $r=r_{ \pm}$, we know that $\Delta(r)=0$, so from above expression of the CME, the numerator of the last term of eq (27) should be zero. Using L'Hospital's rule, we can write

$$
\begin{gathered}
\left.\frac{E_{c m}}{\sqrt{2 m_{1} m_{2}}}\right|_{r \rightarrow r_{ \pm}}=\left[\frac{\left(m_{1}-m_{2}\right)^{2}}{2 m_{1} m_{2}}+\frac{X^{\prime}(r)-\kappa Y^{\prime}(r)}{\Delta^{\prime}(r)}\right]_{r \rightarrow r_{ \pm}}^{\frac{1}{2}} \\
=\left[\frac{\left(m_{1}-m_{2}\right)^{2}}{2 m_{1} m_{2}}+\frac{X^{\prime}\left(r_{ \pm}\right)-\kappa Y^{\prime}\left(r_{ \pm}\right)}{2 r_{ \pm}+r_{\alpha}-2 M}\right]^{\frac{1}{2}}
\end{gathered}
$$

Here $X^{\prime}\left(r_{ \pm}\right)$and $Y^{\prime}\left(r_{ \pm}\right)$are given by

$$
\begin{gathered}
X^{\prime}\left(r_{ \pm}\right)=\left(2 r_{ \pm}+r_{\alpha}-2 M\right)-\frac{2 M L_{1} L_{2}}{\left(r_{ \pm}+r_{\alpha}\right)^{2}}+\frac{2 a M\left(E_{1} L_{2}+E_{2} L_{1}\right)}{\left(r_{ \pm}+r_{\alpha}\right)^{2}}+E_{1} E_{2}\left(2 r_{ \pm}+r_{\alpha}-\frac{2 a^{2} M}{\left(r_{ \pm}+r_{\alpha}\right)^{2}}\right) \\
Y^{\prime}\left(r_{ \pm}\right)=\frac{1}{2} \sqrt{Y_{1}\left(r_{ \pm}\right) Y_{2}\left(r_{ \pm}\right)}\left(\frac{Y_{1}^{\prime}\left(r_{ \pm}\right)}{Y_{1}\left(r_{ \pm}\right)}+\frac{Y_{2}^{\prime}\left(r_{ \pm}\right)}{Y_{2}\left(r_{ \pm}\right)}\right) \\
Y_{i}\left(r_{ \pm}\right)=\left[\left(r_{ \pm}\left(r_{ \pm}+r_{\alpha}\right)+a^{2}+\frac{2 M a^{2}}{r_{ \pm}+r_{\alpha}}\right) E_{i}^{2}-\frac{4 M a}{r_{ \pm}+r_{\alpha}} E_{i} L_{i}\right. \\
\left.-\left(1-\frac{2 M}{r_{ \pm}+r_{\alpha}}\right) L_{i}^{2}-\left(r_{ \pm}\left(r_{ \pm}+r_{\alpha}\right)+a^{2}-2 M r_{ \pm}\right)\right], i=1,2 \\
Y_{i}^{\prime}\left(r_{ \pm}\right)=\left(2 M-2 r_{ \pm}-r_{\alpha}\right)-\frac{2 M L_{i}^{2}}{\left(r_{ \pm}+r_{\alpha}\right)^{2}}+\frac{4 a M E_{i} L_{i}}{\left(r_{ \pm}+r_{\alpha}\right)^{2}}+E_{i}^{2}\left(2 r_{ \pm}+r_{\alpha}-\frac{2 a^{2} M}{\left(r_{ \pm}+r_{\alpha}\right)^{2}}\right), i=1,2
\end{gathered}
$$

\subsection{Collision at extremal horizon}

The extremal horizon (when the event horizon coincides with Cauchy horizon) in Kerr-Sen black hole is located at $r=r_{+}=r_{-}=a=M-\frac{Q^{2}}{M}$. So near the extremal horizon,

$$
\left.\frac{E_{c m}}{\sqrt{2 m_{1} m_{2}}}\right|_{r \rightarrow a}=\left[\frac{\left(m_{1}-m_{2}\right)^{2}}{2 m_{1} m_{2}}+\frac{X^{\prime \prime}(r)-\kappa Y^{\prime \prime}(r)}{\Delta^{\prime \prime}(r)}\right]_{r \rightarrow a}^{\frac{1}{2}}=\left[\frac{\left(m_{1}-m_{2}\right)^{2}}{2 m_{1} m_{2}}+\frac{X^{\prime \prime}(a)-\kappa Y^{\prime \prime}(a)}{2}\right]^{\frac{1}{2}}
$$

Here $X^{\prime \prime}(a)$ and $Y^{\prime \prime}(a)$ are given by

$$
\begin{gathered}
X^{\prime \prime}(a)=2+\frac{4 M L_{1} L_{2}}{\left(a+r_{\alpha}\right)^{3}}-\frac{4 a M\left(E_{1} L_{2}+E_{2} L_{1}\right)}{\left(a+r_{\alpha}\right)^{3}}+E_{1} E_{2}\left(2+\frac{4 a^{2} M}{\left(a+r_{\alpha}\right)^{3}}\right), \\
Y^{\prime \prime}(a)=\frac{1}{4} \sqrt{Y_{1}(a) Y_{2}(a)}\left[2 \frac{Y_{1}^{\prime \prime}(a)}{Y_{1}(a)}+2 \frac{Y_{2}^{\prime \prime}(a)}{Y_{2}(a)}-\left(\frac{Y_{1}^{\prime}(a)}{Y_{1}(a)}-\frac{Y_{2}^{\prime}(a)}{Y_{2}(a)}\right)^{2}\right], \\
Y_{i}(a)=\left[\left(2 a^{2}+a r_{\alpha}+\frac{2 M a^{2}}{a+r_{\alpha}}\right) E_{i}^{2}-\frac{4 M a}{a+r_{\alpha}} E_{i} L_{i}\right.
\end{gathered}
$$




$$
\begin{gathered}
\left.-\left(1-\frac{2 M}{a+r_{\alpha}}\right) L_{i}^{2}-\left(2 a^{2}+a r_{\alpha}-2 M a\right)\right], i=1,2, \\
Y_{i}^{\prime}(a)=\left(2 M-2 a-r_{\alpha}\right)-\frac{2 M L_{i}^{2}}{\left(a+r_{\alpha}\right)^{2}}+\frac{4 a M E_{i} L_{i}}{\left(a+r_{\alpha}\right)^{2}}+E_{i}^{2}\left(2 a+r_{\alpha}-\frac{2 a^{2} M}{\left(a+r_{\alpha}\right)^{2}}\right), i=1,2, \\
Y_{i}^{\prime \prime}(a)=-2+\frac{4 M L_{i}^{2}}{\left(a+r_{\alpha}\right)^{3}}-\frac{8 a M E_{i} L_{i}}{\left(a+r_{\alpha}\right)^{3}}+E_{i}^{2}\left(2+\frac{4 a^{2} M}{\left(a+r_{\alpha}\right)^{3}}\right), i=1,2
\end{gathered}
$$

\subsection{CME near the center of the black hole}

The CME near the center $(r=0)$ of the Kerr-Sen black hole is given by (using equations (27)-(30)

$$
\left.\frac{E_{c m}}{\sqrt{2 m_{1} m_{2}}}\right|_{r \rightarrow 0}=\left[\frac{\left(m_{1}-m_{2}\right)^{2}}{2 m_{1} m_{2}}+\frac{X(0)-\kappa Y(0)}{a^{2}}\right]^{\frac{1}{2}}
$$

where

$$
\begin{gathered}
X(0)=\left[\left(a^{2}+\frac{2 M a}{r_{\alpha}}\right) E_{1} E_{2}-\frac{2 M a}{r_{\alpha}}\left(E_{1} L_{2}+E_{2} L_{1}\right)-\left(1-\frac{2 M}{r_{\alpha}}\right) L_{1} L_{2}+a^{2}\right] \\
Y(0)=\sqrt{Y_{1}(0) Y_{2}(0)}
\end{gathered}
$$

with

$$
Y_{i}(0)=\left[\left(a^{2}+\frac{2 M a}{r_{\alpha}}\right) E_{i}^{2}-\frac{4 M a}{r_{\alpha}} E_{i} L_{i}-\left(1-\frac{2 M}{r_{\alpha}}\right) L_{i}^{2}-a^{2}\right], i=1,2,
$$

We observe that CME $E_{c m}$ for colliding of two particles is finite near the center of the KerrSen black hole. Now if there is no angular momentum (i.e., $a=0$ ) or there is no charge (i.e., $\left.r_{\alpha}=0 \Longrightarrow Q=0\right)$ of the black hole, then we see that the CME $E_{c m}$ diverges near the center of the black hole. But it can be easily determine that $E_{c m} \rightarrow \infty$ at $r \rightarrow \infty$.

\section{Collision of a particle with photon}

Due to the process of Hawking radiation of the black hole, the massless photon coming from the radiation can naturally scatter an infalling particle. This phenomena is analogous to the Compton scattering process. It was originally introduced for a photon and an electron. Now we take into consideration is an infalling particle collides with an outgoing massless photon [52]. Let $U^{a}=\left(U^{t}, U^{r}, U^{\theta}, U^{\phi}\right)$ be the four velocity of the particle. On the equatorial motion of the particle, $\theta=\pi / 2$, so $U^{\theta}=0$. The timelike geodesic of a particle satisfies $g_{a b} U^{a} U^{b}=-1$. So the 4 velocity vectors $U^{t}$ and $U^{\phi}$ along $t$ and $\phi$ directions are same in equations (10) and (11). But the radial component of 4 velocity will be (putting $\epsilon=-1$ in eq (15))

$$
U^{r}= \pm \frac{1}{\sqrt{r\left(r+r_{\alpha}\right)}}\left[A(r) E^{2}-\frac{4 M a}{r+r_{\alpha}} E L-\left(1-\frac{2 M}{r+r_{\alpha}}\right) L^{2}-\Delta(r)\right]^{\frac{1}{2}}
$$

where $E$ and $L$ are respectively the energy and the angular momentum of the particle and $A(r)$ is given in eq (12). Let us assume $K^{a}=\left(K^{t}, K^{r}, K^{\theta}, K^{\phi}\right)$ be the four velocity of the photon. On the equatorial motion of the photon, $\theta=\pi / 2$, so $K^{\theta}=0$. The null geodesic of the photon satisfies [52] 
$g_{a b} K^{a} K^{b}=0$. So the 4 velocity vectors $K^{t}, K^{\phi}$ and $K^{r}$ along $t, \phi$ and $r$ directions are similar to the equations (10), (11) and (15)) (with $\epsilon=0$ ) and given by

$$
\begin{gathered}
K^{t}=\frac{1}{\Delta(r)}\left[A(r) E_{\gamma}-\frac{2 M a}{r+r_{\alpha}} L_{\gamma}\right], \\
K^{\phi}=\frac{1}{\Delta(r)}\left[\frac{2 M a}{r+r_{\alpha}} E_{\gamma}+\left(1-\frac{2 M}{r+r_{\alpha}}\right) L_{\gamma}\right]
\end{gathered}
$$

and

$$
K^{r}= \pm \frac{1}{\sqrt{r\left(r+r_{\alpha}\right)}}\left[A(r) E_{\gamma}^{2}-\frac{4 M a}{r+r_{\alpha}} E_{\gamma} L_{\gamma}-\left(1-\frac{2 M}{r+r_{\alpha}}\right) L_{\gamma}^{2}\right]^{\frac{1}{2}}
$$

where $E_{\gamma}$ and $L_{\gamma}$ are respectively the energy and the angular momentum of the photon. We now determine the center of mass energy (CME) for collision of a particle with rest mass $m$ and a massless photon moving in the equatorial plane. Assume that the four-momentum of the particle is $p^{a}=m U^{a}$ and the photon is $k^{a}=K^{a}(a=t, r, \theta, \phi)$. So the CME of a Hawking photon and the infalling particle can be taken as 52

$$
E_{c m}^{2}=-\left(p^{a}+k^{a}\right)^{2}=m^{2}-2 m g_{a b} U^{a} K^{b}
$$

which yields

$$
E_{c m}^{2}=m^{2}+2 m \frac{P(r)-\kappa Q(r)}{\Delta(r)}
$$

where

$$
\begin{gathered}
P(r)=\left[A(r) E E_{\gamma}-\frac{2 M a}{r+r_{\alpha}}\left(E L_{\gamma}+L E_{\gamma}\right)-\left(1-\frac{2 M}{r+r_{\alpha}}\right) L L_{\gamma}\right], \\
Q(r)=\sqrt{Q_{1}(r) Q_{2}(r)}
\end{gathered}
$$

with

$$
\begin{gathered}
Q_{1}(r)=\left[A(r) E^{2}-\frac{4 M a}{r+r_{\alpha}} E L-\left(1-\frac{2 M}{r+r_{\alpha}}\right) L^{2}-\Delta(r)\right], \\
Q_{2}(r)=\left[A(r) E_{\gamma}^{2}-\frac{4 M a}{r+r_{\alpha}} E_{\gamma} L_{\gamma}-\left(1-\frac{2 M}{r+r_{\alpha}}\right) L_{\gamma}^{2}\right]
\end{gathered}
$$

\section{Photon-photon collision}

Halilsoy and Ovgun [52] stated that the colliding energetic photons in quantum electrodynamics can transmute into particles, so their analysis was entirely classical and hence they have assumed only to the center of mass energy of the yield without further specification. Motivated by their work, here we shall also find the center of mass energy for the collision between two photons in the background of Kerr-Sen black hole. Let us assume $K_{i}^{a}=\left(K_{i}^{t}, K_{i}^{r}, K_{i}^{\theta}, K_{i}^{\phi}\right)(i=1,2)$ be the four velocities of the two photons. On the equatorial motion of the photon, $\theta=\pi / 2$, so $K_{i}^{\theta}=0$. The two photons satisfy the null geodesics in opposite directions. The null geodesic of the two photons satisfy [52] $g_{a b} K_{i}^{a} K_{i}^{b}=0$. So the 4 velocity vectors $K_{i}^{t}, K_{i}^{\phi}$ and $K_{i}^{r}$ along $t, \phi$ and $r$ directions are similar to the equations (47), (48) and (49) and given by

$$
K_{i}^{t}=\frac{1}{\Delta(r)}\left[A(r) E_{\gamma_{i}}-\frac{2 M a}{r+r_{\alpha}} L_{\gamma_{i}}\right]
$$




$$
K_{i}^{\phi}=\frac{1}{\Delta(r)}\left[\frac{2 M a}{r+r_{\alpha}} E_{\gamma_{i}}+\left(1-\frac{2 M}{r+r_{\alpha}}\right) L_{\gamma_{i}}\right]
$$

and

$$
K_{i}^{r}= \pm \frac{1}{\sqrt{r\left(r+r_{\alpha}\right)}}\left[A(r) E_{\gamma_{i}}^{2}-\frac{4 M a}{r+r_{\alpha}} E_{\gamma_{i}} L_{\gamma_{i}}-\left(1-\frac{2 M}{r+r_{\alpha}}\right) L_{\gamma_{i}}^{2}\right]^{\frac{1}{2}}
$$

where $E_{\gamma_{i}}$ and $L_{\gamma_{i}}(i=1,2)$ are respectively the energy and the angular momentum of the $i$-th photon. We now determine the center of mass energy (CME) for collision of two photons moving in the equatorial plane. Assume that the four-momentum of the photons are $k_{i}^{a}=K_{i}^{a}(a=t, r, \theta, \phi$; $i=1,2)$. So the CME of the colliding photons can be taken as 52 .

$$
E_{c m}^{2}=-\left(k_{1}^{a}+k_{2}^{a}\right)^{2}=-2 g_{a b} K_{1}^{a} K_{2}^{b}
$$

which yields

$$
E_{c m}^{2}=2 \frac{Z(r)-\kappa W(r)}{\Delta(r)}
$$

where

$$
\begin{gathered}
Z(r)=\left[A(r) E_{\gamma_{1}} E_{\gamma_{2}}-\frac{2 M a}{r+r_{\alpha}}\left(E_{\gamma_{1}} L_{\gamma_{2}}+E_{\gamma_{2}} L_{\gamma_{1}}\right)-\left(1-\frac{2 M}{r+r_{\alpha}}\right) L_{\gamma_{1}} L_{\gamma_{2}}\right], \\
W(r)=\sqrt{W_{1}(r) W_{2}(r)}
\end{gathered}
$$

with

$$
W_{i}(r)=\left[A(r) E_{\gamma_{i}}^{2}-\frac{4 M a}{r+r_{\alpha}} E_{\gamma_{i}} L_{\gamma_{i}}-\left(1-\frac{2 M}{r+r_{\alpha}}\right) L_{\gamma_{i}}^{2}\right], i=1,2 .
$$

\section{Discussions and Concluding Remarks}

In this work, we have considered static, axially symmetric, rotating and charged Kerr-Sen DilatonAxion black hole metric in generalized Boyer-Lindquist coordinates as particle accelerators. We have obtained the geodesic motions of a particle. We found the effective potential and also found the radius of the circular orbit of a particle. We have investigated the center of mass energy (CME) of two colliding neutral particles (which satisfied timelike geodesic) with different rest masses $m_{1}$ and $m_{2}$ falling from rest at infinity to near the non-extremal horizons (event horizon and Cauchy horizon) and extremal horizon of the Kerr-Sen black hole. We observed that CME $E_{c m}$ for colliding of two particles is finite near the center of the Kerr-Sen black hole. Now if there is no angular momentum (i.e., $a=0$ ) or there is no charge (i.e., $r_{\alpha}=0 \Longrightarrow Q=0$ ) of the black hole, then we have seen that the CME $E_{c m}$ diverges near the center of the black hole. But we found that $E_{c m} \rightarrow \infty$ at $r \rightarrow \infty$.

Analogous to the Compton process, we found the CME for the collision of a particle (which satisfied timelike geodesic) with mass $m$ and a massless photon (which satisfied null geodesic) where photons can be coming from the Hawking radiation of the black hole. Finally, we found the CME due to the collision of two photons (which satisfied null geodesic) in the background of Kerr-Sen black hole.

Acknowledgement: The author is thankful to IUCAA, Pune, India for warm hospitality where the work was carried out. 


\section{References}

[1] M. Bañados, J. Silk and S. M. West, Phys. Rev. Lett. 103, 111102 (2009).

[2] K. Lake, Phys. Rev. Lett. 104, 211102 (2010).

[3] S. W. Wei, Y. X. Liu, H. Guo and C.-E. Fu, Phys. Rev. D 82, 103005 (2010).

[4] T. Harada and M. Kimura, Classical Quantum Gravity 31, 243001 (2014).

[5] C. Liu, S. Chen, C. Ding and J. Jing, Phys. Lett. B 701, 285 (2011).

[6] A. Zakria, M. Jamil, JHEP 1505 (2015) 147.

[7] M. Sharif and N. Haider, J. Theor. Exp. Phys. 117, 78 (2013).

[8] P. Pradhan, arXiv:1407.0877 [gr-qc].

[9] J. L. Said and K. Z. Adami, Phys. Rev. D 83, 104047 (2011).

[10] G. Abbas U. Sabiullah, Astrophys. Space Sci. 352, 769 (2014).

[11] C. Bambi and L. Modesto, Phys. Lett. B 721, 329 (2013).

[12] P. Pradhan, arXiv:1402.2748 [gr-qc].

[13] N. Haider, Open J. Mod. Phys. 1, 1 (2014).

[14] M. Amir, S.G. Ghosh, arXiv:1503.08553 [gr-qc].

[15] S. G. Ghosh and M. Amir, arXiv: 1506.04382 [gr-qc].

[16] B. Pourhassan and U. Debnath, arXiv:1506.03443 [gr-qc].

[17] A. Tursunov, M. Kolo.s, A. Abdujabbarov, B. Ahmedov and Z. Stuchlik, Phys. Rev. D 88, 124001 (2013).

[18] S. Fernando, Gen. Rel. Grav. 46, 1634 (2014).

[19] T. Jacobson, T. P. Sotiriou, Phys. Rev. Lett. 104, 021101 (2010).

[20] E. Berti, V. Cardoso, L. Gualtieri, F. Pretorius, U. Sperhake, Phys. Rev. Lett. 103, 239001 (2009).

[21] M. Banados, B. Hassanain, J. Silk and S. M. West, Phys. Rev. D 83, 023004 (2011).

[22] T. Harada, M. Kimura, Phys. Rev. D 83, 024002 (2011).

[23] M. Patil, P. S. Joshi and D. Malafarina, Phys. Rev. D 83, 064007 (2011).

[24] M. Patil and P. S. Joshi, Phys. Rev. D 82, 104049 (2010).

[25] M. Patil, P. S. Joshi, M. Kimura and K. I. Nakao, Phys. Rev. D 86, 084023 (2012).

[26] M. Patil and P. S. Joshi, Classical Quantum Gravity 28, 235012 (2011). 
[27] T. Igata, T. Harada and M. Kimura, Phys. Rev. D 85, 104028 (2012).

[28] I. Hussain, Mod. Phys. Lett. A 27, 1250017 (2012).

[29] M. Sharif and N. Haider, Astrophys. Space Sci. 346, 111 (2013).

[30] O. B. Zaslavskii, JETP Lett. 92, 571 (2010).

[31] S. W. Wei, Y. X. Liu, H. T. Li and F. W. Chen, JHEP 12, 066 (2010).

[32] J. Sadeghi, B. Pourhassan, Eur. Phys. J. C. 72, 1984 (2012).

[33] J. Sadeghi, B. Pourhassan, H. Farahani, Commun. Theor. Phys. 62, 358 (2014).

[34] A. A. Grib, Yu.V. Pavlov, Astropart. Phys. 34, 581 (2011).

[35] S. G. Ghosh, P. Sheoran, M. Amir, Phys. Rev. D 90 (2014) 103006.

[36] M. Sharif and N. Haider, Astrophys. Space Sci. 346, 111 (2013).

[37] G. N. Gyulchev and S. S. Yazadjiev, Phys. Rev. D 81, 023005 (2010).

[38] A. Sen, Phys. Rev. Lett. 69, 1006 (1992).

[39] A. Larranaga, Pramana 76, 553 (2011).

[40] P. Pradhan, arXiv: 1503.04514[gr-qc].

[41] G. N. Gyulchev and S. S. Yazadjiev, AIP Conf. Proc. 1301, 327 (2010).

[42] K. Hioki and U. Miyamoto, Phys. Rev. D 78, 044007 (2008).

[43] Q. Q. Jiang, S. -Z. Yang, D. -Y. Chen, Chin. Phys. 15, 1709 (2006).

[44] G. N. Gyulchev, S. S. Yazadjiev, Phys. Rev. D 75, 023006 (2007).

[45] G. -Q. Li, Theor. Math. Phys. 153, 1652 (2007).

[46] S. -Z. Yang, D. -Y. Chen, Chin. Phys. Lett. 24, 39 (2007).

[47] G. N. Gyulchev, S. S. Yazadjiev, AIP Conf. Proc. 899, 145 (2007).

[48] D. -Y. Chen, X. -T. Zu, Mod. Phys. Lett. A 24, 1159 (2009).

[49] A. Larranaga, Pramana 76, 553 (2011).

[50] P. Pradhan, arXiv:1503.04514,

[51] F. Khani, M. T. Darvishi, R. Baghbani, Astrophys. Space Sci. 350, 275 (2014).

[52] M. Halilsoy and A. Ovgun, arXiv:1507.00633[gr-qc]. 